\title{
The study protocol for PREDICT AF RECURRENCE: a PRospEctive cohort stuDy of survelllanCe for perioperaTive Atrial Fibrillation RECURRENCE in major non-cardiac surgery for malignancy
}

Satoshi Higuchi ${ }^{1,10^{*}} \mathbb{D}$, Yusuke Kabeya ${ }^{2,3}$, Kenichi Matsushita' $^{1}$ Keisei Tachibana ${ }^{4}$, Riken Kawachi ${ }^{5}$, Hidefumi Takei ${ }^{4}$, Yutaka Suzuki ${ }^{6}$, Nobutsugu Abe ${ }^{6}$, Yorihisa Imanishi ${ }^{7}$, Kiyoshi Moriyama ${ }^{8}$, Tomoko Yorozu ${ }^{8}$, Koichiro Saito ${ }^{9}$, Masanori Sugiyama ${ }^{6}$, Haruhiko Kondo ${ }^{4}$ and Hideaki Yoshino ${ }^{1}$

\begin{abstract}
Background: A previous retrospective cohort study established the relationship between perioperative atrial fibrillation (POAF) and subsequent mortality and stroke. However, the details regarding the cause of death and etiology of stroke remain unclear.
\end{abstract}

Methods: The prospective cohort study of surveillance for perioperative atrial fibrillation recurrence in major noncardiac surgery for malignancy (PREDICT AF RECURRENCE) registry is an ongoing prospective cohort study to elucidate the long-term recurrence rate and the clinical impact of new-onset POAF in the setting of head and neck, non-cardiac thoracic, and abdominal surgery for malignancy. In this study, cardiologists collaborate with a surgical team during the perioperative period, carefully observe the electrocardiogram (ECG) monitor, and treat arrhythmia as required. Furthermore, patients who develop new-onset POAF are followed up using a long-term Holter ECG monitor, SPIDER FLASH-t AFib', to assess POAF recurrence.

Discussion: Even if patients with malignancy survive by overcoming the disease, they may die from any preventable cardiovascular diseases. In particular, those with POAF may develop cardiogenic stroke in the future. Because details of the natural history of patients with POAF remain unclear, investigating the need to continue anticoagulation therapy for such patients is necessary. This study will provide essential information on the recurrence rate of POAF and new insights into the prediction and treatment of POAF.

Trial registration: University Hospital Medical Information Network Clinical Trial Registry (UMIN-CTR): UMIN000016146; Data of Registration: January 7, 2015.

Keywords: Perioperative atrial fibrillation (POAF), Non-cardiac surgery, Oncology, Stroke

\footnotetext{
*Correspondence: sahiguchi-circ@umin.ac.jp

'Division of Cardiology, Department of Internal Medicine II, Kyorin University

School of Medicine, Tokyo, Japan

${ }^{10}$ Division of Cardiology, Department of Internal Medicine II, Kyorin University

School of Medicine, 6-20-2 Shinkawa, Mitaka City, Tokyo 181-8611, Japan

Full list of author information is available at the end of the article
}

(c) The Author(s). 2018 Open Access This article is distributed under the terms of the Creative Commons Attribution 4.0 International License (http://creativecommons.org/licenses/by/4.0/), which permits unrestricted use, distribution, and reproduction in any medium, provided you give appropriate credit to the original author(s) and the source, provide a link to the Creative Commons license, and indicate if changes were made. The Creative Commons Public Domain Dedication waiver (http://creativecommons.org/publicdomain/zero/1.0/) applies to the data made available in this article, unless otherwise stated. 


\section{Background}

Atrial fibrillation (AF) is one of the most common types of arrhythmia and a common health-related problem, with increasing incidence and prevalence worldwide [1, 2]. AF is associated with increased mortality and incidence of thromboembolic events, such as stroke [3-5], and is most likely induced by clinical conditions, such as hypertension, diabetes mellitus, and heart failure [6-8]. Furthermore, AF often occurs after both cardiac and non-cardiac surgeries, usually on the second or third postoperative day [9], with an incidence rate of $10-65 \%$ and $1-9 \%$, respectively [9-11]. The massive differences in the reported incidence rate could be attributed to the possible overlooking of short-duration AF and the variable impact of each surgical procedure on the incidence. Although perioperative AF (POAF) seems to be a temporary cardiac event that does not affect the subsequent clinical course, POAF is reported to be associated with long-term mortality and stroke, even in the setting of non-cardiac surgery and general clinical situations [10]. Furthermore, some recent studies demonstrated that malignancy was related to a higher prevalence of AF [12-14]. Notably, Conen et al. demonstrated that the multi-variate adjusted hazard ratio of AF after diagnosis of breast cancer was as high as 4.67 (95\% CI, 2.85-7.64) in the first 3 months, regardless of treatment assignments [14].

Despite recognizing AF as an independent risk factor for mortality, the evidence to determine AF as a causal factor is insufficient [15]. Hence, determining whether $\mathrm{AF}$ is the direct and decisive cause of death in the general population without any cardiac disease remains doubtful. In cardiac surgery, the frequent occurrence of POAF with impaired cardiac function might induce cardiac events, such as heart failure and cardiac death. However, in non-cardiac surgery, wherein patients do not usually present with such impairment whether AF directly correlates with mortality or only a surrogate marker remains undetermined.

The recent advancements in the treatment of malignancies have enhanced the prognosis of such patients, increasing the subsequent survivor population at the risk of cardiovascular events [16]. Patients with AF recurrence after the perioperative period are susceptible to subsequent embolic events; therefore, both long- and short-term POAF management to prevent adverse clinical events should be considered. Although a previous study [10] suggested that anticoagulation therapy could be beneficial for patients with POAF, it did not identify patients who were at high risk for subsequent stroke and who should start anticoagulation therapy after discharge. If most factors causing stroke are related to atherosclerosis, antiplatelet therapy along with appropriate risk management regimen should be provided. Particularly, patients with malignancy sometimes face complicated treatment choices because of elevated thrombotic and bleeding risks [17]. In such cases, a survey detailing the short- and long-term clinical course of those with POAF might be helpful for subsequent daily clinical management.

\section{Hypothesis}

The present study has been conducted to investigate our primary hypothesis that the patients with new-onset POAF will develop AF recurrence in the future.

\section{Methods/design \\ Study design and population}

The PRospEctive cohort stuDy of surveIllanCe for perioperaTive Atrial Fibrillation RECURRENCE (PREDICT AF RECURRENCE) in major non-cardiac surgery for malignancy is an ongoing prospective, single-center, observational study that is designed to illustrate the clinical impact of POAF on mortality and morbidity as cardiologists collaborate with a surgical team during the perioperative period and investigate the frequency of AF recurrence after discharge in patients with malignancy. In this study, we examined consecutive patients who underwent non-cardiac surgery under general anesthesia due to definitive or suspected malignancy. We followed patients who developed new-onset AF by using a cardiac event recorder. Figure 1 shows the flowchart of the study. The present study enrolled from July 2014 and the registered patients will be followed up until December 2022.

\section{Inclusion criteria}

In this study, we enrolled patients (age: $\geq 20$ and $\leq 90$ years) who planned to undergo surgery in Kyorin University Hospital (Tokyo, Japan) from July 2014. The malignancies registered were as follows: head and neck (e.g., pharyngeal, laryngeal, tongue, mandible, buccal mucosal, gingival, and glottic), chest (e.g., esophageal, lung, and lung metastases), or abdomen (gallbladder, extra- and intrahepatic bile duct, pancreatic, duodenal, Vater papillary, hepatic cell, and liver metastases) cancers. Some previous studies have demonstrated that the prevalence of POAF was frequently associated with the surgery site and, thus, could be infrequent in less-invasive surgical treatments $[10,18]$. Hence, we did not include patients with gastric and colon cancers in this study because a majority of them were treated with less-invasive laparoscopic surgery in our institute.

\section{Exclusion criteria}

In this study, we excluded patients with persistent or chronic AF and atrial flutter. Although those with known paroxysmal AF before surgery were included for in-hospital analysis, they were excluded in the analysis of long-term AF recurrence. 


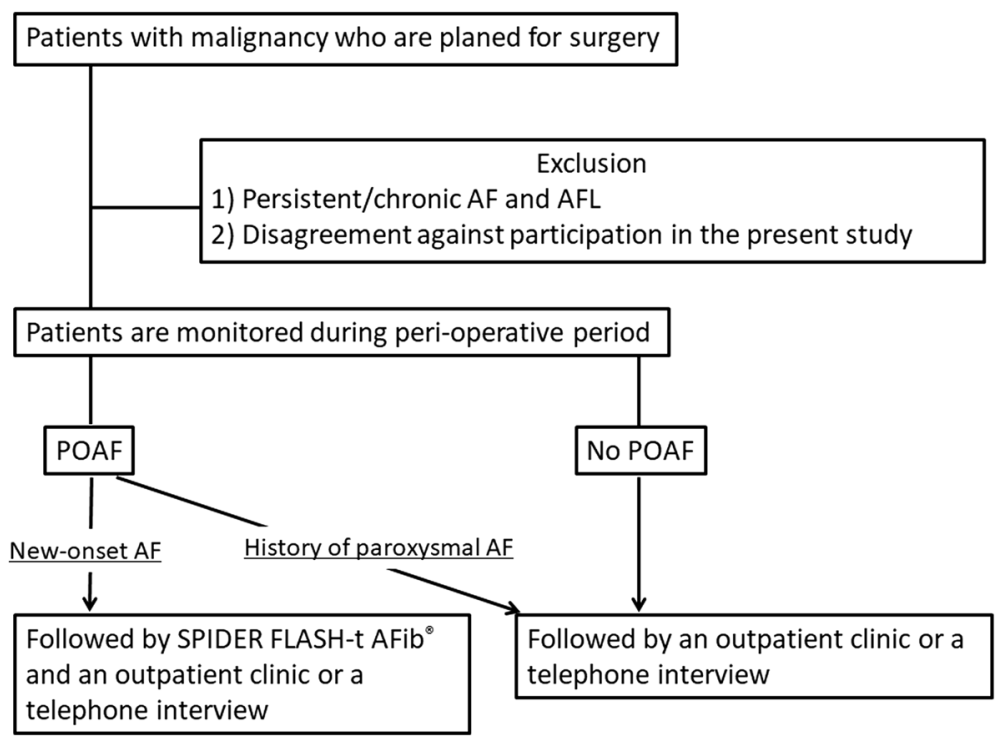

Fig. 1 The flowchart of PREDICT AF RECURRENCE. Patients with new-onset AF are followed by using SPIDER FLASH-t AFib ${ }^{\oplus}$. AF Atrial fibrillation, AFL Atrial flutter, POAF Perioperative atrial fibrillation

\section{Baseline assessment}

The baseline survey was conducted 1-3 days preoperatively by using a study-specific questionnaire for collecting information, including age, sex, current medications, alcohol consumption, smoking status, past histories of thoracotomy and cardiogenic stroke, and complications, such as heart failure, coronary artery disease, peripheral artery diseases, and chronic obstructive pulmonary disease. Table 1 shows the definition of each complication. We performed blood examination within 3 months preoperatively, which included blood cell counts, C-reactive protein (CRP), D-dimer, liver enzyme, electrolytes, serum creatinine, thyroid hormone, and B-type natriuretic peptide (BNP). In addition, we performed a respiratory function test (including forced expiratory volume and forced vital capacity) and 12-lead electrocardiography (ECG) at the same time with blood examination. As a routine clinical practice in the institute, Doppler ultrasonography was performed on patients with D-dimer level of $\geq 0.5 \mu \mathrm{g} / \mathrm{mL}$ to assess superficial and deep venous thromboses of the lower extremities.

\section{Data collection during general anesthesia}

During the surgery, anesthesiologists provided general care to patients. In addition, heart rate, rhythm, and ST-T changes were continuously monitored by an ECG and appropriately recorded. The final diagnosis of each arrhythmia was confirmed by cardiologists.

\section{Operation record}

We recorded the stage of malignancy, type of surgery, extent of tumor resection and lymph node dissection,
Table 1 Definition of complications

\begin{tabular}{|c|c|}
\hline \multirow[t]{3}{*}{ Heart Failure } & Fulfills any of the following: \\
\hline & $\begin{array}{l}\text { 1) Any symptoms such as orthopnea, } \\
\text { paroxysmal nocturnal dyspnea, or dyspnea } \\
\text { on effort and BNP } \geq 100 \mathrm{pg} / \mathrm{mL}\end{array}$ \\
\hline & $\begin{array}{l}\text { 2) A history of admission because of } \\
\text { congestive heart failure. }\end{array}$ \\
\hline Hypertension & $\begin{array}{l}\text { Systolic blood pressure } \geq 140 \mathrm{mmHg} \text { and/or } \\
\text { diastolic blood pressure } \geq 90 \mathrm{mmHg}\end{array}$ \\
\hline \multirow[t]{3}{*}{ Diabetes Mellitus } & Fulfills 1) and 2) \\
\hline & $\begin{array}{l}\text { 1) Fasting plasma glucose } \geq 126 \mathrm{mg} / \mathrm{dL} \text { or } \\
\text { casual plasma glucose } \geq 200 \mathrm{mg} / \mathrm{dL}\end{array}$ \\
\hline & 2) $\mathrm{HbA} 1 \mathrm{c} \geq 6.5 \%$ \\
\hline Coronary Artery Disease & $\begin{array}{l}\text { Patients with any of the following: } L A D, L C X \text {, } \\
\text { or RCA with stenosis } \geq 75 \% \text {, or LMT with } \\
\text { stenosis } \geq 50 \% \text { if a patient has undergone } \\
\text { coronary angiography or computed } \\
\text { tomography. }\end{array}$ \\
\hline Peripheral Artery Disease & $\begin{array}{l}\text { The term includes carotid artery stenosis/ } \\
\text { occlusion, intracranial middle- and large- } \\
\text { artery stenosis/occlusion, renal stenosis, } \\
\text { arteriosclerosis obliterans, and aortic } \\
\text { aneurysm, all of which are diagnosed by any } \\
\text { imaging modality. }\end{array}$ \\
\hline \multirow{3}{*}{$\begin{array}{l}\text { Chronic Obstructive } \\
\text { Pulmonary Disease }\end{array}$} & fulfills 1) and 2) \\
\hline & 1) $\mathrm{FEV}_{1} / \mathrm{FVC}$ ratio $<0.7$ \\
\hline & $\begin{array}{l}\text { 2) Predicted to be irreversible after the } \\
\text { administration of an inhaled } \\
\text { bronchodilator }\end{array}$ \\
\hline
\end{tabular}

BNP, B-type natriuretic peptide; $L A D$, left anterior descending artery; $L C x$, left circumflex artery; RCA, right coronary artery; LMT, left main trunk; FEV , forced expiratory volume in 1 second; FVC, forced vital capacity 
and pathological findings in the patients. The stage and extent of lymph node dissection in each surgery were determined based on the Union of International Cancer Control (UICC) TNM classification seventh edition.

\section{Postoperative data collection}

In this study, all patients were observed with an ECG monitor device for a minimum of $24 \mathrm{~h}$ up to 30 days postoperatively; the monitoring duration was based on each surgeon's discretion. In addition, duration of monitoring and surgical intensive care unit stay were recorded. Patients with any type of arrhythmia requiring an intervention were treated by cardiologists in accordance with the current guideline. Furthermore, anticoagulation therapy was provided for those who developed AF if CHA2DS2 VASc score [19] was $\geq 1$ and $\geq 2$ in men and women, respectively, after surgeons assessed the bleeding risk and permitted the prescription. Subsequently, the presence of symptoms, onset time/day, and total duration of AF during hospitalization were recorded, and blood examination, such as white blood cell, hemoglobin, serum creatinine, CRP, and electrolytes, was planned on the following and the fourth postoperative day. We examined the presence of postoperative complications, including myocardial infarction; congestive heart failure; bleeding; thrombosis, such as stroke, transient ischemic attack, ischemic bowel disease, and pulmonary embolism; any infections; acute kidney injury; and desaturation necessary for home oxygen therapy. Finally, echocardiography was immediately performed for patients with new-onset AF.

\section{Follow-up after discharge}

In this ongoing study, the follow-up data would be collected from a face-to-face interview in an outpatient clinic, a telephone interview, or a medical record. The follow-up flow chart is shown in Table 2 . Regardless of the presence of POAF, the patients in this study would be followed up for all-cause, cardiac, non-cardiac, and malignancy-related mortality; AF occurrence or recurrence; and stroke. The diagnosis and etiology of stroke in this study were determined by two or more neurologists and radiologists independent from the investigators. The patients who developed new-onset AF were continuously followed up using SPIDER FLASH-t AFib (LivaNova, London, UK) for approximately 2 weeks after 1 and 12 months postoperatively. The device can record heart rhythm non-invasively for the longest duration in Japan. The dynamic memory in the device allows for rhythm episodes to be accurately identified regardless of their duration. Any arrhythmia, including AF, is recorded automatically (Additional file 1: Table S1). In addition, follow-up was performed after 6 months if required.
Table 2 Follow-up in chronological order

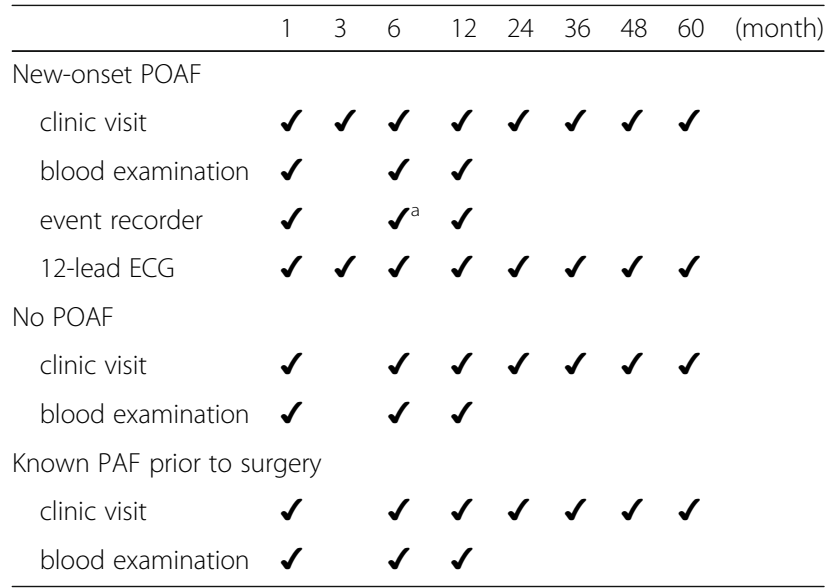

$a_{\text {: }}$ if required

ECG: electrocardiogram; PAF: paroxysmal atrial fibrillation; POAF: perioperative atrial fibrillation

Follow-up was recorded throughout the day except during the time when patients were taking a bath. Patients who were unable to handle SPIDER FLASH-t $\mathrm{AFib}^{\circ}$ were examined using 24-h Holter ECG for 2 to 4 times. If $\mathrm{AF}$ recurrence was documented 1 month postoperatively, the following event monitoring was not planned. The patients who developed a rash because of an electrode seal at the first record could stop the following examination. If the patients were hospitalized at 1,6 , or 12 months postoperatively, they could be evaluated using an ECG monitor device of the residing ward instead of SPIDER FLASH-t AFib. The records acquired by SPIDER FLASH-t AFib were assessed by an external expert organization, and the final decision was confirmed by two cardiologists. With regard to the 24-h Holter ECG and ECG monitor device in the ward, two cardiologists analyzed and diagnosed the recorded ECG. In the case of differential diagnosis, they attain consensus after sufficient discussion to conclude the final diagnosis. Information on anticoagulation, such as type, dose, duration, and adherence, are collected by cardiologists. Of note, patients without AF recurrence for a year can discontinue anticoagulation treatment.

\section{Outcome definition}

Detailed outcomes defined in Additional file 2.

\section{Short-term outcome}

In this study, short-term outcomes comprise new-onset POAF; any arrhythmia; embolic events, such as stroke; and mortality until the thirtieth day after surgery. We defined POAF as AF during and/or after surgery until the thirtieth postoperative day. In addition, we also evaluated the type of arrhythmia, etiology of stroke, and cause of death. 


\section{Long-term outcome}

We followed up all patients with and without POAF in this study. Long-term outcomes included AF recurrence (evaluated only in those with new-onset POAF); embolic events, such as stroke; recurrent or de novo malignancy; and mortality on and after the thirty-first postoperative day. New-onset AF occurring on and after the thirty-first day was not considered as POAF, but as "conventional" paroxysmal AF. We defined AF recurrence as repetitive AF lasting $\geq 30 \mathrm{~s}$ on and after the thirty-first day, which was documented by SPIDER FLASH-t AFib ${ }^{\circ}$, 24-h Holter ECG, or hospital ECG monitor [20]. Notably, patient's self-assessment and symptoms did not contribute to the definitive diagnosis. Furthermore, the cause of death included cardiac, non-cardiac, and cancer-related mortalities.

\section{Sub-analysis}

We will conduct sub-analyses investigating the predictor of POAF in each malignancy, and the incidence and the characteristics of other arrhythmias.

\section{Statistical analysis}

No preceding study evaluated the incidence of AF recurrence in patients with POAF. Therefore, we assumed the incidence in reference to the first 200 patietns in our study, which indicated a $25 \%$ recurrence rate. We assumed a $0.97 \%$ incidence of $\mathrm{AF}$ in patients without POAF based on a previous study [6]. Based on $80 \%$ power and a significance level of $0.05,38$ patients with new-onset POAF were required. Assuming a $15 \%$ dropout rate and a $9 \%$ incidence rate of POAF, 497 (new-onset POAF, $n=45$; non-POAF, $n=452$ ) were required at least. In this study, the relationship between exposure variables and in-hospital outcomes was assessed using logistic regression analyses and expressed as odds ratio, 95\% confidential interval, and $P$ value. Variables with $P<0.10$ in the univariate logistic regression analysis were included in the multivariate logistic regression analysis. In addition, we assessed the long-term outcomes using the survival analysis methods. The cumulative incidence of long-term outcomes was assessed using the Kaplan-Meier statistics. Furthermore, the hazard ratios of long-term outcomes were calculated using the Cox regression analysis.

\section{Discussion}

To the best of our knowledge, this study is the first research to prospectively investigate the long-term recurrence and the clinical impact of POAF using an event recorder in patients with malignancy.

Early documentation of POAF could be essential to prevent potential embolic events. In addition, underdiagnoses are often possible because AF lasts for a very short period, and an ECG monitor is the only means to provide a definitive diagnosis. To date, several risk scores and equations for the prediction of $\mathrm{AF}$ in the general population have been developed and validated. In this study, we will validate the efficacy of such risk scores in the setting of the perioperative period by assessing our dataset.

Even if patients with malignancy survive by overcoming the disease, they might die from any preventable cardiovascular diseases. In particular, those with POAF might develop cardiogenic stroke in the future. Because details of the natural history of patients with POAF remain unclear, investigating the need to continue anticoagulation therapy for such patients is necessary.

The apparent correlation between POAF and stroke can be explained by other atherosclerotic factors, such as age, hypertension, and diabetes mellitus $[6,7,21]$. Therefore, whether AF causes subsequent stroke remains unclear. Physicians in this study follow up patients in real time and record the incidence of POAF and any complications, which might be helpful for assuming the causal relationship between AF and morbidities, chronologically. Furthermore, the instrumental variable method, if appropriate, could reinforce the supposed correlation.

Although POAF post-non-cardiac surgery is an increasingly common problem and attracts clinicians' attention, both short- and long-term characteristics and management have remained unclear because of limited evidence [22].

This study has some limitations. First, the present cohort incudes limited subsets of non-cardiac surgery. The results acquired from the present study may not be adapted for the other subsets, such as non-malignancy. Second, the incidence of AF in the patietns without POAF may be underestimated because they do not wear the event recorder. However, the most important question is whether we should prescribe anticoagulation for patients with POAF in future. We believe that the results of this cohort will provide several indications for the management of patients with malignancy who developed POAF.

\section{Additional files}

$$
\text { Additional file 1: Table S1. The settings of automatic detection }
$$

Additional file 2: "Definition of outcome" and "Details of SPIDER FLASH-t AFib". (DOCX 19 kb)

\section{Abbreviations}

AF: Atrial fibrillation; ECG: Electrocardiogram; POAF: Perioperative atrial fibrillation 


\section{Consent to publication}

Not applicable.

\section{Funding}

This study is funded by JSPS KAKENHI Grant Number 17 K18085.

\section{Authors' contributions}

SH designed the study and drafted manuscript. YK, KM, KT, RK, HT, YS, NA, YI, $\mathrm{KM}, \mathrm{TY}, \mathrm{KS}, \mathrm{MS}, \mathrm{HK}$, and $\mathrm{HY}$ designed the study and revised the manuscript critically for important intellectual content. Final approval of the manuscript submitted was done by $\mathrm{SH}$.

\section{Ethics approval and consent to participate}

This study protocol conforms to the ethical guidelines of the 1975 Declaration of Helsinki in line with the Ethical Guidelines for Epidemiological Research by the Japanese government. This study was approved by the ethics committee of Kyorin University Hospital (approval number: 488) and registered on University Medical Information Network (UMIN ID: UMIN000016146). Written informed consent was obtained from patients before each surgery by the physicians.

\section{Competing interests}

The authors declare that they have no competing interests.

\section{Publisher's Note}

Springer Nature remains neutral with regard to jurisdictional claims in published maps and institutional affiliations.

\section{Author details}

'Division of Cardiology, Department of Internal Medicine II, Kyorin University School of Medicine, Tokyo, Japan. ${ }^{2}$ Division of General Internal Medicine, Department of Internal Medicine, Tokai University, Isehara, Kanagawa, Japan. ${ }^{3}$ Department of Home Care Medicine, Saiyu Clinic, Saitama, Japan. ${ }^{4}$ Department of General Thoracic Surgery, Kyorin University School of Medicine, Tokyo, Japan. ${ }^{5}$ Department of General Thoracic Surgery, Nihon University School of Medicine, Tokyo, Japan. ${ }^{6}$ Department of Surgery, Kyorin University School of Medicine, Tokyo, Japan. ${ }^{7}$ Department of Otorhinolaryngology, Head and Neck Surgery, Kawasaki Municipal Kawasaki Hospital, Kawasaki, Kanagawa, Japan. ${ }^{8}$ Department of Anesthesiology, Kyorin University School of Medicine, Tokyo, Japan. ${ }^{9}$ Department of Otolaryngology-Head and Neck Surgery, Kyorin University School of Medicine, Tokyo, Japan. ${ }^{10}$ Division of Cardiology, Department of Internal Medicine II, Kyorin University School of Medicine, 6-20-2 Shinkawa, Mitaka City, Tokyo 181-8611, Japan.

Received: 5 April 2018 Accepted: 11 June 2018

Published online: 26 June 2018

\section{References}

1. Lip GYH, Brechin CM, Lane DA. The global burden of atrial fibrillation and stroke: a systematic review of the epidemiology of atrial fibrillation in regions outside North America and Europe. Chest. 2012;142(6):1489-98.

2. Chugh SS, Havmoeller R, Narayanan $K$, Singh D, Rienstra M, Benjamin EJ Gillum RF, Kim YH, McAnulty JH Jr, Zheng ZJ, et al. Worldwide epidemiology of atrial fibrillation: a global burden of disease 2010 study. Circulation. 2014;129(8):837-47.

3. Benjamin EJ, Wolf PA, D'Agostino RB, Silbershatz H, Kannel WB, Levy D. Impact of atrial fibrillation on the risk of death: the Framingham heart study. Circulation. 1998;98(10):946-52.

4. Chugh SS, Blackshear JL, Shen WK, Hammill SC, Gersh BJ. Epidemiology and natural history of atrial fibrillation: clinical implications. J Am Coll Cardiol. 2001:37(2):371-8.

5. Patel NJ, Deshmukh A, Pant S, Singh V, Patel N, Arora S, Shah N, Chothani A, Savani GT, Mehta K, et al. Contemporary trends of hospitalization for atrial fibrillation in the United States, 2000 through 2010: implications for healthcare planning. Circulation. 2014;129(23):2371-9.

6. Krahn AD, Manfreda J, Tate RB, Mathewson FA, Cuddy TE. The natural history of atrial fibrillation: incidence, risk factors, and prognosis in the Manitoba follow-up study. Am J Med. 1995;98(5):476-84.
7. Benjamin EJ, Levy D, Vaziri SM, D'Agostino RB, Belanger AJ, Wolf PA. Independent risk factors for atrial fibrillation in a population-based cohort. The Framingham Heart Study. JAMA. 1994;271(11):840-4.

8. Santhanakrishnan R, Wang N, Larson MG, Magnani JW, McManus DD, Lubitz SA, Ellinor PT, Cheng S, Vasan RS, Lee DS, et al. Atrial fibrillation begets heart failure and vice versa: temporal associations and differences in preserved versus reduced ejection fraction. Circulation. 2016;133(5):484-92.

9. Maisel WH, Rawn JD, Stevenson WG. Atrial fibrillation after cardiac surgery. Ann Intern Med. 2001;135(12):1061-73.

10. Gialdini G, Nearing K, Bhave PD, Bonuccelli U, ladecola C, Healey JS, Kamel H. Perioperative atrial fibrillation and the long-term risk of ischemic stroke. JAMA. 2014;312(6):616-22.

11. Batra GS, Molyneux J, Scott NA. Colorectal patients and cardiac arrhythmias detected on the surgical high dependency unit. Ann R Coll Surg Engl. 2001; 83(3):174-6.

12. O'Neal WT, Lakoski SG, Qureshi W, Judd SE, Howard G, Howard VJ, Cushman M, Soliman EZ. Relation between cancer and atrial fibrillation (from the REasons for geographic and racial differences in stroke study). Am J Cardiol. 2015;115(8):1090-4.

13. Erichsen R, Christiansen CF, Mehnert F, Weiss NS, Baron JA, Sorensen HT Colorectal cancer and risk of atrial fibrillation and flutter: a population-based case-control study. Intern Emerg Med. 2012;7(5):431-8.

14. Conen D, Wong JA, Sandhu RK, Cook NR, Lee IM, Buring JE, Albert CM. Risk of malignant Cancer among women with new-onset atrial fibrillation. JAMA Cardiol. 2016;1 (4):389-96.

15. Leong DP, Eikelboom JW, Healey JS, Connolly SJ. Atrial fibrillation is associated with increased mortality: causation or association? Eur Heart J. 2013;34(14):1027-30.

16. Chung R, Maulik A, Hamarneh A, Hochhauser D, Hausenloy DJ, Walker JM, Yellon DM. Effect of remote Ischaemic conditioning in oncology patients undergoing chemotherapy: rationale and design of the ERIC-ONC study-a single-center, blinded, randomized controlled trial. Clin Cardiol. 2016;39(2): $72-82$.

17. Melloni C, Shrader P, Carver J, Piccini JP, Thomas L, Fonarow GC, Ansell J, Gersh B, Go AS, Hylek E, et al. Management and outcomes of patients with atrial fibrillation and a history of cancer: the ORBIT-AF registry. Eur Heart J Qual Care Clin Outcomes. 2017:3(3):192-7.

18. Bhave PD, Goldman LE, Vittinghoff E, Maselli J, Auerbach A. Incidence, predictors, and outcomes associated with postoperative atrial fibrillation after major noncardiac surgery. Am Heart J. 2012;164(6):918-24.

19. Lip GY, Nieuwlaat R, Pisters R, Lane DA, Crijns HJ. Refining clinical risk stratification for predicting stroke and thromboembolism in atrial fibrillation using a novel risk factor-based approach: the euro heart survey on atrial fibrillation. Chest. 2010;137(2):263-72

20. Barthelemy JC, Feasson-Gerard S, Garnier P, Gaspoz JM, Da Costa A, Michel $D$, Roche F. Automatic cardiac event recorders reveal paroxysmal atrial fibrillation after unexplained strokes or transient ischemic attacks. Ann Noninvasive Flectrocardiol. 2003:8(3):194-9.

21. Majeed A, Moser K, Carroll K. Trends in the prevalence and management of atrial fibrillation in general practice in England and Wales, 1994-1998: analysis of data from the general practice research database. Heart. 2001; 86(3):284-8.

22. Vallurupalli S, Shanbhag A, Mehta JL. Controversies in postoperative atrial fibrillation after noncardiothoracic surgery: clinical and research implications. Clin Cardiol. 2017:40(5):329-32.

\section{Ready to submit your research? Choose BMC and benefit from}

- fast, convenient online submission

- thorough peer review by experienced researchers in your field

- rapid publication on acceptance

- support for research data, including large and complex data types

- gold Open Access which fosters wider collaboration and increased citations

- maximum visibility for your research: over $100 \mathrm{M}$ website views per year

\section{At BMC, research is always in progress.}

Learn more biomedcentral.com/submissions 